\title{
Linx
}

Revue des linguistes de l'université Paris X Nanterre

70-71| 2014

Variations sémantiques et syntaxiques : aspects d'une théorie de l'invariance

\section{ENTRE, entre préposition et préfixe}

\section{Fumitake Ashino}

\section{(2) OpenEdition \\ Journals}

Édition électronique

URL : http://journals.openedition.org/linx/1573

DOI : $10.4000 /$ linx.1573

ISSN : 2118-9692

Éditeur

Presses universitaires de Paris Nanterre

\section{Édition imprimée}

Date de publication : 1 septembre 2014

Pagination : 125-141

ISSN : 0246-8743

\section{Référence électronique}

Fumitake Ashino, «ENTRE, entre préposition et préfixe », Linx [En ligne], 70-71 | 2014, mis en ligne le 01 mars 2015, consulté le 06 mai 2019. URL : http://journals.openedition.org/linx/1573 ; DOI : 10.4000/ linx. 1573 


\section{ENTRE, entre préposition et préfixe}

Fumitake Ashino, Université Keio, Tokyo

\section{Introduction $^{1}$}

La réflexion sur la façon dont s'articulent invariance et variation des unités morpho-lexicales est au cœur du programme de recherches du groupe de travail Théorie des Opérations Prédicatives et Enonciatives (TOPE). Différents travaux inscrits dans ce programme de recherche ont été menés, notamment dans le cadre des études sur la polysémie verbale (Saunier 1996 ; Paillard 2000 ; Camus \& De Vogüé 2004 ; Franckel \& Paillard 2007 ; Pauly 2010).

La thèse défendue dans cette approche consiste à postuler que l'invariance d'une unité morpho-lexicale (verbe, nom, adjectif, préposition, préfixe, mot du discours, etc.) ne peut être pensée que par la prise en compte de sa variation (ses différents emplois et valeurs) ; autrement dit, c'est précisément dans la mesure où une telle unité varie que son invariance peut être (re)construite.

Dans cet article, nous voulons contribuer à la réflexion sur l'articulation invariance / variation des unités morpho-lexicales non seulement en relation avec la question de la polysémie, mais aussi et surtout en relation avec les catégories syntaxico-morphologiques.

En français comme dans d'autres langues, nombreuses sont les unités qui ont plusieurs appartenances catégorielles. Tel est le cas de l'unité entre(-) que nous étudions ici.

Nous tenons à remercier Sarah de Vogüé, ainsi que le relecteur anonyme pour leurs remarques et critiques qui ont permis de préciser certains points de cet article. 
En français contemporain, entre(-) fonctionne aussi bien comme préposition que comme préfixe. Il n'est pas question ici d'étudier l'ensemble des problèmes que pose l'articulation de ces deux catégories, mais d'examiner un cas précis de concurrence, où entre préposition et entre- préfixe (verbe préfixé en entre-) apparaissent intuitivement comme très proches, comme le montre la paire d'exemples suivante :

(a) Ils se tuent entre eux.

(b) Ils s'entre-tuent.

Nous appelons construction prépositionnelle la construction en (a), où entre forme un GP avec un (pro)nom au pluriel, et construction préfixale la construction en (b), où l'on a un verbe préfixé en entre-. L'un et l'autre exemples expriment par ailleurs la réciprocité.

Notre objectif consiste à montrer que l'unité entre, selon les constructions dans lesquelles elle s'inscrit, ne fonctionne pas de la même façon : chaque construction est soumise à des contraintes syntaxiques différentes et supporte des effets de sens particuliers. Nous limitons notre discussion à l'étude de ces deux constructions avec le verbe pronominal se tuer.

\section{Problématique}

\section{1. Étude de Van Goethem (2009)}

Différents travaux portent sur l'unité entre (Franckel \& Paillard 2007 ; Rivière \& Guentchéva 2007 pour entre préposition ; Benetti \& Heyna 2006 ; Van Goethem 2009 pour entre(-) préfixe / préposition). Ci-dessous nous discutons l'ouvrage de Van Goethem (2009) qui constitue l'étude la plus récente sur la question de l'articulation préfixe / préposition : sur(-), contre(-) et entre(-).

L'auteure considère le préfixe (ou "préverbe» dans sa terminologie) comme «un emploi de la préposition» (cf. le titre de son ouvrage : L'emploi préverbal des prépositions en français). Inscrit dans le cadre de la théorie de la grammaticalisation, cet ouvrage se propose d'« étudier les préverbes dans leurs différents degrés de préfixation" pour examiner «comment les prépositions sont susceptibles de subir un processus de grammaticalisation et de se transformer en "véritables préfixes" ".

L'hypothèse de départ sur les prépositions consiste à les décrire comme ayant une double incidence, portant sur deux objets syntaxiques : «cible» et « site», terminologie que l'auteure emprunte à l'étude de Vandeloise (1995) sur les prépositions du français.

Pour mettre en évidence le processus de grammaticalisation, elle s'appuie sur différents paramètres morpho-syntaxiques et sémantiques.

A) Le paramètre morpho-syntaxique permet de distinguer trois grandes classes de constructions préverbales en référence à la relation qui peut être établie entre le préfixe et la préposition :

(i) Constructions «relationnelles »: le préverbe a un fonctionnement analogue à celui de la préposition correspondante ; 
(ii) Constructions «préfixales » : le préverbe s'éloigne de son origine prépositionnelle et se comporte comme un préfixe incident à sa base ;

(iii) Constructions «lexicalisées »: le rapport entre le préverbe et la préposition n'est plus transparent.

Ces trois classes de constructions sont illustrées respectivement par les trois exemples suivants qui mettent en jeu le préverbe sur-:

(1) L'avion survole les îles Baléares.

(2) Il a surestimé ses capacités.

(3) surjaler $^{2}$

En (1), le préverbe sur-porte sur le GN les îles Baléares (" site ») ; l'avion (" cible ») est mis en relation avec ce GN par le biais du préverbe sur. En (2), en revanche, l'auteure considère le préverbe sur-, "plus grammaticalisé », comme n'ayant pas de portée syntaxique, mais comme étant incident à la base (estimer), et indiquant «l'excès de confiance en soi »; en (3), le verbe surjaler correspond au cas où il n'y a pas de rapport transparent entre le préverbe et sa portée.

Concernant le préfixe entre- que nous étudions, Van Goethem fait l'hypothèse qu'il relève principalement des constructions préverbales relationnelles de type (i) ${ }^{3}$, où le préverbe, comme son correspondant prépositionnel, se combine avec une base verbale et porte sur un objet nominal, souvent implicite.

Deux cas de figure sont distingués. Le premier cas concerne les exemples dans lesquels entre- exprime une "relation de réciprocité entre les participants »: dans ce groupe d'exemples, le préverbe entre- se combine avec des verbes pronominaux :

(4) Elle était contente que Gaspard fût là, lui, la meilleure tête de la famille. Quand ils s'entre-regardaient, elle éprouvait que l'amitié demeurait et malgré tout une confiance. (TLF, cité par Van Goethem 2009, p.145)

Van Goethem fait l'hypothèse que le préverbe entre- dans cet exemple se rapporte au pronom se dont le référent peut être explicité. Ce qui conduit l'auteure à considérer l'exemple (4') ci-dessous comme la paraphrase de l'exemple (4) :

(4') [...] Quand ils se regardaient entre eux elle éprouvait que l'amitié demeurait et malgré tout une confiance.

Le deuxième cas, illustré par l'exemple (5), concerne des emplois dans lesquels le préverbe entre- apparaît avec des verbes non pronominaux, exprimant alors ce qu'elle décrit comme «l'interruption (multiple) du référent de l'objet» :

(5) De grands sanglots entrecoupaient ses phrases. (TLF, cité par V. Goethem 2009, p. 146)

2 Selon TLFi, ce verbe signifie : «être engagé sous le jas et faire un tour par-dessus (en parlant de la chaîne de l'ancre) ». construction.

Selon l'auteure, près de $60 \%$ des emplois du préverbe entre- correspondent à cette 
Dans cet exemple, le préverbe entre-indique, selon l'auteure, qu'un élément («le site ॥) rendu par l'objet direct est interrompu à plusieurs reprises par un autre élément (« la cible »), ici par le sujet.

B) Le deuxième paramètre ("paramètre sémantique ») se présente comme l'autre façon de mesurer le degré de grammaticalisation de entre-, selon que le préfixe partage ou non ses « emplois » avec ceux de la préposition.

Les emplois du préfixe entre- sont décrits sur la base d'une combinaison des trois traits sémantiques : [intermédiaire], [bipolaire] et [réciproque], qui sont les mêmes traits qui caractérisent entre préposition. C'est la présence ou l'absence de ces traits (marqués respectivement par + et -) qui caractérise chaque emploi.

Trois grandes classes d'emplois sont ainsi distinguées : (i) «emplois spatiaux»; (ii) « emplois aspectuels »; (iii) « emplois notionnels » :

(i) entretoiser, entrecouper [+ intermédiaire, + bipolaire, - réciproque]

(ii) $\left(s^{\prime}\right)$ entrouvrir, $\left(s^{\prime}\right)$ entretenir [+ intermédiaire, + bipolaire, - réciproque]

(iii) s'entre-tuer [-intermédiaire, +/- bipolaire, +réciproque]

On voit que le verbe s'entre-tuer qui nous intéresse est classé dans le troisième emploi dit « notionnel».

De ce qui précède, l'auteure conclut que le préfixe entre-, tant sur les plans morpho-syntaxique que sémantique, est proche de son correspondant prépositionnel, et donc que c'est un cas de faible grammaticalisation.

\subsection{Différences syntactico-sémantiques entre les constructions prépositionnelle et préfixale}

La démonstration par Van Goethem du caractère relationnel du préfixe dans les cas de valeur réciproque se fonde sur un rapprochement entre la construction prépositionnelle et la construction préfixale considérées comme équivalentes (cf. la mise en relation entre (4) et (4')). Cependant, on observe des différences syntacticosémantiques entre ces deux formes se manifestant notamment à travers les différences d'acceptabilité : la forme prépositionnelle paraît d'un emploi très contraint.

Sur le plan syntaxique, on observe plusieurs différences relatives aux contraintes distributionnelles qui pèsent sur la construction prépositionnelle (et non sur la construction préfixale). Les deux types de contraintes ci-dessous concernent l'un et l'autre la nature du sujet syntaxique :

(i) La construction prépositionnelle est bloquée avec certains types de $\mathbf{N}$ en position de sujet (tous (6) et on (7)) :

(6) Je lisais avec la petite veilleuse de mon siège ce récit avec une acuité que j'avais rarement eue. Il m'a bouleversé dans ce qu'il avait d'innocent. Aucun personnage qui ne croie à l'enchantement du monde ! Tous veulent s'entre-tuer \{?? se tuer entre eux\}, mais aucun ne doute que le monde soit enchanté. (Le Monde)

(7) Soudain, tout le monde se tait. « Si on parle politique, on va finir par s'entre-tuer $\left\{{ }^{*}\right.$ se tuer entre nous\} ", confie une mère de famille, en chuchotant à l'écart de la foule. (Libération) 
(ii) La construction prépositionnelle est contrainte, lorsque le terme en position de sujet est désigné par un GN duel constitué de deux $\mathbf{N}$ coordonnés ((8), (9)) ou quantifié par le numéral deux (10):

(8) Suivant les consignes, ils ne doivent «pas intervenir à chaud sur les Postiches » afin de ne pas provoquer de grabuge avec les otages, mais les prendre en filature et les arrêter plus tard. Las, l'impulsif commissaire Raymond Mertz, patron de la BRB, fait feu, ce qui déclenche une fusillade : un gangster et un policier s'entre-tuent \{?? se tuent entre eux $\}$. Deux braqueurs s'enfuient avec des flics en otages. (Libération)

(9) Durant le siège, Polynice et Etéocle s'entre-tuèrent \{?? se tuèrent entre eux , concrétisant ainsi la malédiction lancée par leur père. (Webcorp)

(10) Palestiniens et Israéliens sont deux frères siamois qui s'entre-tuent \{?? se tuent entre eux\}. (Benetti \& Heyna 2006, p.618)

Sur le plan sémantique, il existe des effets de sens particuliers associés à chacune des constructions :

(iii) La construction préfixale (et non la construction prépositionnelle) permet une interprétation " métaphorique " de (se) tuer :

(11) Ce jour-là, Britney Spears vient à Paris assurer la promo de son premier « film », Crossroads. [...] Après le photo call, anglicisme désignant une meute de photographes qui s'entre-tuent \{?? se tuent entre eux \} pour shooter l'artiste à tir tendu pendant une poignée de minutes, vient la conférence de presse [...]. (Libération)

(12) La première chose à noter, c'est qu'il n'y a pas d'édition d'Angoulême sans scandale, arias comminatoires ou, plus généralement, chantage affectif. Les critiques, auteurs et éditeurs s'entre-tuent \{?? se tuent entre eux $\}$ généralement sur la sélection, jugée tour à tour trop grand public ou trop pointue. (Libération)

(iv) La construction prépositionnelle apparaît comme "délimitant un groupe d'individus" ou comme "opposant un groupe d'individus à un autre " (Rivière \& Guentcheva 20074) :

(13) Malheureusement, il arrive souvent que les chasseurs se tuent entre eux.

(14) Le narcotrafic a gagné des batailles culturelles et médiatiques dans une société qui s'est protégée du problème en ayant recours au déni : «Les tueurs se tuent entre eux ». (Courrier International)

(v) s'entre-tuer n'est pas un verbe d'achèvement à la différence de se tuer entre eux :

(15) a. Ils se sont entretués pendant des heures.

b. *Ils se sont tués entre eux pendant des heures.

4 «It [ = entre eux $]$ does not express a reciprocal meaning on its own, but often limits or emphasizes the group of persons involved in a reciprocal, or non-reciprocal situation, by sometimes opposing them to other people or presenting the action as a characteristic of these people » (Rivière \& Guentchéva 2007, p. 591). Cette description nous semble pertinente, mais les auteures n'expliquent pas d'où vient cet effet de sens. Nous y reviendrons lorsque nous étudions la préposition entre. 
Compte tenu de ces différences syntactico-sémantiques entre les constructions prépositionnelle et préfixale, on ne saurait les considérer comme des équivalentes, en tout cas en synchronie. La question se pose donc de savoir comment arriver à les distinguer et à rendre compte de leurs différences, tout en prenant en considération la continuité entre les deux constructions. Cela revient donc à articuler le même (invariance) et le différent (variation).

L'approche de Van Goethem supposerait que le garant du même soit la préposition, plus précisément ses propriétés morpho-syntaxiques (ses points d'«incidence ») et sémantiques («ses emplois»), dont l'emploi préfixal serait un cas plus ou moins éloigné (" grammaticalisé »). Dans cette perspective, le différent (variation) n'est envisageable que de façon négative, correspondant en l'occurrence au fait pour le préfixe de ne pas vérifier les propriétés de la préposition, conçue comme étant son origine.

Dans l'approche que nous proposons, c'est l'invariant sémantique commun aux deux constructions qui constitue ce même qui les rassemble, un invariant qui constitue l'identité sémantique de entre, et que nous formulons à l'aide d'une forme schématique 5 restant stable quel que soit le statut de entre; quant au different, il tient aux façons différentes dont cette forme schématique se redéploie dans l'espace référentiel de l'énoncé et notamment à la façon dont elle se combine avec celle du verbe en jeu. Cela nous permet de donner un statut propre à entre préposition et entre- préfixe en tant qu'ils entrent dans deux structures distinctes, au lieu de décrire le préfixe comme un dérivé de la préposition plus ou moins grammaticalisé.

Ces structures étant liées à la relation qui s'établit avec le verbe, les paragraphes qui suivent sont consacrés aux verbes et à leurs modes de fonctionnement spécifiques, avec d'abord une brève présentation des propriétés sémantiques et syntaxiques qui leur sont propres, puis une ébauche de caractérisation du verbe tuer pris en exemple et de son fonctionnement avec le réfléchi se.

\section{Trois niveaux de représentation d'un verbe}

À la suite de De Vogüé (2004) et de Franckel \& Paillard (2007), nous considérons que la description d'un verbe implique de distinguer entre trois plans : (i) celui de la relation prédicative qui s'établit au niveau de la proposition et qui est spécifique aux verbes; (ii) celui de la Forme Schématique qui définit l'identité sémantique du verbe en tant qu'item lexical; (iii) celui du (ou des) schémas syntaxiques effectifs dans lesquels le verbe peut apparaître en tant que forme réalisée, dotée de différents arguments ('argument sujet d'une part, mais aussi tous les compléments réalisés, tous placés ici au même niveau comme étant tous les éléments qui viennent compléter le verbe).

Les paragraphes qui suivent sont consacrés à la mise en évidence de chacun de ces trois plans, puis à l'illustration de la façon dont ils se trouvent articulés dans le cas du verbe tuer.

\footnotetext{
$5 \quad$ On rappelle ici la façon dont Culioli introduit la notion de forme schématique : «L'analyse d'un marqueur (entendu non pas au sens d'étiquette, mais de marqueur d'opération ou éventuellement de polyopération) doit aboutir à une représentation formelle ayant des caractères stables et contrôlables. À partir de cette représentation formelle, que j’appelle forme schématique, se construisent des formes supplémentaires qui sont, en fait, des déformations de la forme de base. La question est de comprendre l'organisation de ces dispositifs déformables ». (Culioli 1990, p.116)
} 
ENTRE, entre préposition et préfixe

\section{(i) Relation prédicative}

La relation prédicative est le premier plan de représentation d'un verbe comme prédicat. Elle est donnée sous la forme d'un schéma prédicatif avec une structure argumentale minimale du type 'source' / 'but', représentée sous la forme suivante: $<() \mathrm{r}()>$, où la place de gauche correspond à la source, la place de droite au but. Le terme « source » peut se trouver instancié par un terme en position thématique, ce qui peut correspondre au GN en position sujet : c'est sur ce plan que le sujet se voit par conséquent distingué par rapport aux autres compléments du schéma syntaxique. Cependant, la relation prédicative est une représentation abstraite du prédicat et, à ce titre, elle est en deçà des termes se trouvant effectivement instanciés : source et but peuvent ne pas être instanciés du tout ${ }^{6}$.

\section{(ii) Forme schématique (FS)}

La FS d'un verbe correspond à une représentation sémantique du verbe définissant son identité sémantique en tant qu'unité lexicale. Une FS met en jeu des paramètres sémantiques abstraits (ou éléments de la FS) notés $a, b, c .$. Ces paramètres sémantiques sont là aussi en deçà d'une assignation lexicale (qu'à la suite de Paillard (2007), nous appelons lexicalisation) et sont susceptibles de faire l'objet de multiples spécifications.

\section{(iii) Schéma syntaxique}

Un verbe s'inscrit par ailleurs dans un schéma syntaxique du type Co $\mathrm{V} \mathrm{C}_{1}$ $\mathrm{C}_{2} \ldots \mathrm{C}_{\mathrm{n}}$, où Co correspond au sujet syntaxique, $\mathrm{C}_{1}$ au complément d'objet direct, $\mathrm{C}_{2}$ au complément second éventuel, $\mathrm{C}_{3} \ldots \mathrm{C}_{\mathrm{n}}$ aux différents compléments indirects possibles. Une unité lexicale instanciant l'un des paramètres (ou éléments) de la FS du verbe peut occuper différentes positions dans ce schéma, et correspondre par conséquent à différentes fonctions syntaxiques selon les cas.

Pour rendre compte du fait qu'un même verbe peut entrer dans différentes constructions, il est nécessaire de se donner ce double plan de caractérisation (ii)-(iii). Pour expliquer qu'une même base lexicale peut prendre la forme d'un verbe ou d'une autre catégorie syntaxique, il est nécessaire de se donner en outre le plan (i), en tant que pouvant être distinct de (ii) et de (iii).

On sort ainsi d'une perspective strictement distributionnelle caractérisant les unités par leurs seuls compléments instanciés : il devient possible de distinguer entre les arguments sémantiques que mobilise le verbe, les arguments instanciés qui décrivent sa distribution, et les arguments prédicatifs qui peuvent ne s'aligner ni sur la structure sémantique, ni sur le schéma syntaxique (quand la source n'est ni le premier des arguments sémantiques, ni le complément Co dit sujet). Cela devient non seulement possible mais aussi nécessaire : pour caractériser l'emploi d'un verbe, on doit pouvoir spécifier ses arguments sémantiques, ses compléments, et la structure prédicative dans laquelle il s'intègre.

\footnotetext{
Le terme source pourra être autre chose qu'un GN, notamment dans les propositions sans
sujet. Par exemple, dans une proposition impersonnelle comme $I /$ pleut, le terme source pourra être la situation dont on prédique cette caractérisation que constitue la présence de pluie.
} 


\section{Se tuer}

Nous illustrons ci-dessous la façon dont ces trois plans s'articulent dans le cas du verbe tuer (3.1.) et de sa forme pronominale se tuer (3.2.).

\subsection{FS de tuer}

Faute d'une forme schématique un tant soit peu stabilisée pour le verbe tuer, nous nous contentons ici d'une ébauche qui vise simplement à déployer les différents paramètres que ce verbe mobilise :

- une « entité » $(b)$ est prise dans le passage d'un « état initial » $\left(e_{1}\right)$ à un « état autre » $\left(e_{2}\right)$, qui est, à des degrés divers, la négation de $e_{1}$;

- un « déclencheur » (a) est à l'origine de ce passage.

Commentaires :

- A noter que la notion de 'mort' que l'on serait tenté d'associer à tuer n'est pas constitutive de ce qui fait l'identité sémantique du verbe: elle n'est qu'une des interprétations possibles de ce que recouvre le passage en question, selon les propriétés lexicales des $\mathrm{N}$ correspondant à l'élément $a$ et à l'élément $b$;

- Il y a une altérité forte entre $e_{1}$ et $e_{2}$ ( $e_{2}$ étant défini comme la négation de $e_{1}$ ), ce qui tend à conférer au verbe tuer sa dimension « télique $»^{7}$;

- Cette altérité repose sur une dissymétrie entre une position associée au bénéfactif et l'autre position qui se trouve associée au détrimental.

Cette ébauche de FS de tuer peut être illustrée par les trois exemples suivants :

(16) Paul a tué sa femme.

(17) Tu m'as tué!

(18) Paul flâne pour tuer le temps.

En (16), tuer marque que sa femme lexicalisant l' « entité » (b) de la FS passe de $e_{1}$ ('être en vie') à $e_{2}$ ('trépas'). Paul correspond à l'élément $a$ ("déclencheur ») et, à ce titre, il est l'origine de la mort de sa femme. Nous insistons sur le fait que l'interprétation de cet énoncé comme décrivant la mort découle d'une configuration particulière où le « déclencheur » et l'« entité » sont tous les deux lexicalisés par un $\mathrm{N}$ du type /humain/. Mais cette configuration ne garantit pas toujours cette interprétation. En effet, en (17), bien que les deux $\mathrm{N}$ lexicalisant respectivement $a$ et $b$ soient du type /humain/ (tu et $m e$ ), $e_{2}$ ne s'interprète pas comme la 'mort' de me (b) (a priori, ce dernier peut difficilement se dire 'mort'), mais comme son 'épuisement' dont le déclencheur est tu (a) ; $e_{1}$ est donc interprétable comme 'être dans un état normal'.

En (18), enfin, on a une tout autre interprétation. L'élément $b$ est lexicalisé par le temps, mais relativement à $\mathrm{Paul}^{8}$, ce qui suppose une mise en relation de Paul et du temps. e s'interprète donc comme le fait que 'Paul dispose de temps'. Mais, à la

\footnotetext{
7 Toutefois, nous verrons que la télicité a priori inscrite dans le verbe tuer peut ne pas être pertinente lorsque celui-ci est préfixé en entre-.

$8 \quad$ Cela vient du fait que le temps est inséparable d'un support de sa manifestation.
} 
différence des cas de passer le temps et de perdre son temps, ce temps dont Paul dispose a une valeur détrimentale (le temps à éliminer, à faire passer), associée à un ennui, à une attente sans finalité, etc., en somme tout ce qui échappe à tout investissement et/ou contrôle du sujet. Quant à $e_{2}$, il peut être interprété comme 'le temps éliminé'. Par ailleurs, à cause de la construction en pour, a ne correspond pas à Paul, mais à la 'flânerie', en l'occurrence au fait que 'Paul flâne' : il s'agit à nouveau d'un terme mettant en relation deux éléments lexicaux dans l'énoncé, Paul et flâne.

\subsection{Se tuer}

Pour analyser la forme pronominale se tuer, nous proposons de décrire se comme un marqueur d'opération intervenant au niveau de la relation prédicative en relation avec les éléments de la FS :

se marque que l'un des éléments de la FS du verbe n'est pas lexicalisé, mais est récupérable par mise en relation avec le terme source de la relation prédicative9.

Nous montrons comment cette hypothèse sur se se combine avec le verbe tuer dont la FS est proposée ci-dessus.

(19) Paul s'est tué d'une balle de revolver.

(20) Paul s'est tué dans un accident de voiture.

(21) Paul se tue au travail.

En (19), l'élément $a$ (" déclencheur») est lexicalisé par Paul, plus précisément 'Paul utilisant le revolver'; l'élément $b$ (« entité ») n'est pas lexicalisé, mais se pose qu'il est récupérable à partir de Paul, terme source. L'interprétation de cet énoncé comme un 'suicide' vient du fait que d'une part, Paul a un double statut ("déclencheur» et «entité ») et que d'autre part, il utilise un instrument, ce qui lui confère un statut d'agent intentionnel. Paul comme « entité » est pris dans le passage de $e_{1}$ ('être en vie') à $e_{2}$ ('être mort'); Paul est lui-même le « déclencheur » de ce passage.

En (20), l'élément $a$ correspond à Paul, plus précisément 'Paul impliqué dans un accident de voiture' ou 'Paul dans la voiture'. L'élément $b$ non lexicalisé est récupéré par le terme source Paul. À la différence de (19), l'accident de voiture étant un événement qui échappe au contrôle du sujet, Paul ne saurait être considéré comme 'agent'.

En (21), le mécanisme est comparable. L'élément $b$ non lexicalisé est récupéré à partir de Paul (plus précisément 'Paul travaillant' ou 'Paul au travail') qui correspond à l'élément $a$ et qui est donné comme détrimental. $e_{1}$ et $e_{2}$ s'interprètent respectivement comme 'être en forme / en bonne santé' et 'dégradation de cet état initial'.

\section{Se tuer entre eux comme « fermeture du procès "}

\footnotetext{
$9 \quad$ Cette hypothèse est une reformulation de la caractérisation du postfixe sja en russe proposée par D. Paillard, en y introduisant l'articulation du niveau de la relation prédicative avec le niveau de la forme schématique : «Nous définissons sja comme la trace d'une opération prédicative, qui consiste à «vider » une des places de la relation prédicative, qui, par là même, devient réflexive ». (Paillard 1979, p. 76).
} 


\subsection{FS de entre}

Pour ce qui est de l'identité sémantique de entre, nous nous appuyons ci-dessous sur l'étude que consacrent Franckel \& Paillard (2007) à cette préposition et sur la Forme Schématique qu'ils proposent pour en rendre compte. Dans leur ouvrage, la préposition entre, comme toutes les autres prépositions, est définie comme étant un 'relateur' du type $\mathbf{X} \mathbf{R} \mathbf{Y}$ où $\mathbf{R}$ correspond à la préposition, et où $\mathbf{Y}$ correspond au terme introduit par la préposition; $\mathbf{X}$ en revanche est variable, et peut correspondre à un autre terme de l'énoncé, ou à tout autre élément correspondant à des paramètres non nécessairement lexicalisés des formes schématiques en présence. Le rapport entre $\mathbf{X}$ et $\mathbf{Y}$ n'est pas symétrique : $\mathbf{Y}$ est défini comme repère de $\mathbf{X}$ (terme repéré) et, à ce titre, $\mathbf{Y}$ est source de détermination pour $\mathbf{X}$.

Plus précisément, dans le cas de entre (et aussi de l'ensemble des prépositions analysées dans le volume de Franckel \& Paillard (2007) $)^{10}$, Y s'entend comme un domaine par rapport auquel $\mathbf{X}$ se trouve repéré, la notion de domaine correspondant ici à ce qu'Antoine Culioli a proposé pour rendre compte des différents repérages possibles d'une occurrence par rapport à une notion, distinguant différentes zones dans le domaine (un intérieur $\mathbf{I}$, un extérieur $\mathbf{E}$, mais aussi des frontières variées structurées sur une base topologique). On peut alors distinguer entre différents repérages possibles pour les prépositions, selon que $\mathbf{X}$ se trouve repéré dans l'intérieur $\mathbf{I}$ de ce domaine ou au contraire dans son extérieur $\mathbf{E}$, dans différentes formes de zones frontières, et selon aussi que l'intérieur $\mathbf{I}$ est mis en opposition à $\mathbf{E}$, ou est donné comme une zone indéterminée, sans prise en compte d'éventuelles zones extérieures, ou avec des prises en compte variables.

Nous résumons ci-dessous la FS de entre que proposent Franckel \& Paillard (2007, p. 40):

Étant donné l'altérité première entre les deux zones $\mathbf{I}$ et $\mathbf{E}$ sur le domaine associé à $\mathbf{Y}$, entre marque que cette altérité (notée $\mathbf{I} \mid \mathbf{E}$ ) est neutralisée (notée I-E).

Selon leur analyse, entre s'interprète par conséquent comme marquant une neutralisation de l'altérité première entre $\mathbf{I}$ et $\mathbf{E}$ sur le domaine associé à $\mathbf{Y}$ par la mise en relation de $\mathbf{X}$ avec ce domaine.

On voit que par rapport aux trois traits distingués par Van Goethem, la bipolarité se retrouve dans l'opposition entre $\mathbf{I}$ et $\mathbf{E}$, et le trait $+/$ - intermédiaire (mais aussi les cas où on a le trait - bipolaire) peut être mis en relation avec la neutralisation dont il est ici question. Mais l'idée est de ne pas se contenter de juxtaposer ces trois traits en les autorisant à être plus ou moins présents, mais de donner les paramètres constitutifs d'entre dans tous ses emplois et de calculer comment ces paramètres vont pouvoir donner différentes valeurs, dont des valeurs plus ou moins bipolaires, des valeurs pouvant plus ou moins s'entendre en termes d'intermédiaire et des valeurs plus ou moins réciproques.

10 Il s'agit des prépositions dites de « division », prépositions « qui associent à $\mathbf{Y}$ un domaine qu'elles divisent et configurent, chacune de façon particulière» (Franckel \& Paillard 2007, p. 215). Elles s'opposent à celles dites de " discernement », où «Y attribue des propriétés non définitoires au terme $\mathbf{X}$ mis en relation à $\mathbf{Y}$ par la préposition ». (ibid, p. 8) 
Par ailleurs, dans une telle approche, on ne considère pas qu'il existerait en soi des catégories pré-établies - catégories de l'intermédiaire, ou du réciproque : on dispose de paramètres à partir desquels ces catégories doivent être construites.

\subsection{Trois types de neutralisation}

La variation des valeurs rencontrées pour entre est rapportée au fait que la neutralisation I-E va pouvoir s'interpréter de trois façons différentes, que l'on se propose d'illustrer par les trois exemples ci-dessous :

(22) Il devra choisir entre plusieurs solutions.

(23) Marie est assise entre Paul et Jacques.

(24) Malheureusement, il arrive souvent que les chasseurs se tuent entre eux.

À chaque fois, la façon dont $\mathbf{I}$ se trouve interprété dépend du prédicat en jeu, ce qui signifie que la valeur obtenue pour entre va dépendre crucialement de la nature de la prédication effectuée : c'est celle-ci qui détermine le domaine $\mathbf{Y}$ et ce qui peut figurer l'intérieur de ce domaine.

(i) La neutralisation (I-E) s'interprète comme une indétermination de la valeur à retenir

Cette forme de neutralisation se traduit par le fait qu'on est en attente d'une telle valeur. Ainsi, en (22), plusieurs solutions correspond à $\mathbf{Y} ; \mathbf{X}$, quant à lui, n'est pas lexicalisé, mais est interprétable, en relation avec le verbe choisir, comme correspondant à 'une solution à choisir'. L'altérité première entre $\mathbf{I}$ et $\mathbf{E}$ peut donc s'interpréter comme celle entre 'une solution (effectivement) sélectionnée' (I) et 'les autres solutions qui ne sont pas sélectionnées' (E). Cette altérité est d'ordre virtuel, dans la mesure où l'on est en deçà de savoir quelle solution va être effectivement sélectionnée. La neutralisation I-E signifie donc qu'on ne peut pas encore distinguer le sélectionné (I) du non sélectionné (E) : les solutions relevant respectivement de $\mathbf{I}$ et de $\mathbf{E}$ sont sur le même plan, en tant que « sélectionnables »; en ce sens elles sont neutralisées, parce que le choix de I reste indéterminé.

(ii) La neutralisation s'interprète comme caractérisant une zone qui peut être rapportée à la fois à $I$ et à $E$

Dans ce cas de figure, illustré par l'exemple (23), l'altérité première s'interprète comme celle que fondent deux positions distinctes, soit $\mathbf{I}$ et $\mathbf{E}$, pour ce qui est de la localisation de $\mathbf{X}$. Y correspond à Paul et Jacques. Dans le cadre de la localisation de Marie (X), Paul et Jacques sont interprétables respectivement comme $\mathbf{I}$ et $\mathbf{E}$, deux positions distinctes qui sont, chacune, susceptibles de localiser Marie ('Marie est à côté de Paul (I)' ou 'Marie est à côté de Jacques $\left.(\mathbf{E})^{\prime}\right)$. Dire qu'il y a neutralisation de ces deux positions (I-E) signifie que pour ce qui est de la localisation de Marie, et Paul et Jacques sont le localisateur de Marie; autrement dit, Marie est à la fois dans l'espace de Paul et dans celui de Jacques. D'où l'interprétation de « position intermédiaire ».

On voit qu'au lieu que la valeur «intermédiaire» soit donnée comme caractéristique d'entre, elle est donnée comme le résultat de l'association de cette préposition avec un prédicat de localisation (ici être assis; ailleurs conper, parce que l'on coupe 'quelque part', dans des énoncés comme Il a coupé entre les deux nouds). 


\section{(iii) La neutralisation s'interprète comme une identification de $E$ à $I$}

Ce cas de figure correspond à la construction prépositionnelle mettant en jeu entre qui nous intéresse. Dans l'exemple (24), le GN en Co (les chasseurs) correspond à $\mathbf{X}$, le GN en $\mathrm{C}_{2}(e u x)$ à $\mathbf{Y}$. L'altérité première ici s'interprète comme une opposition / un contraste, sur un mode effectif ou fictif, entre un ensemble (I) et un terme extérieur (E) qui a / aurait un rapport avec I dans le cadre du procès désigné par le verbe. Sans cette opposition préalable, ce type d'énoncés devient contraint, comme le montre le contraste d'acceptabilité dans les énoncés suivants :

(25) a. ? Ils se saluent entre eux.

b. Ils ne se saluent qu'entre eux.

c. Ils se saluent toujours entre eux.

d. Ils se saluent entre camarades.

Pour ne prendre que (25b), ne...que marque le mouvement qui fait passer des cas où le procès désigné par saluer peut concerner d'autres termes que ceux de l'ensemble (cf. ils saluent les autres) à une configuration où ce procès concerne uniquement cet ensemble.

En (24), ce contraste est exprimé par la séquence malheureusement, il arrive souvent... : I (ensemble) correspond au GN en Co, soit les chasseurs; $\mathbf{E}$ (terme extérieur) quant à lui n'est pas lexicalisé, mais compte tenu de la FS du verbe et de son rapport avec les chasseurs, il est reconstituable comme ce qui fait l'objet d'une chasse, donc un $\mathrm{N}$ désignant a priori un 'gibier'. La neutralisation signifie que $\mathbf{E}$, a priori posé comme extérieur à I, ne l'est en fait pas, E étant identifié à I (il n'y a pas de $\mathbf{E}$ en dehors de I) : de ce fait, $\mathbf{I}$ se trouve caractérisé comme un ensemble «fermé » relatif au procès. Ce qui explique que dans ce cas de figure, il y a coréférence entre le terme en Co (les chasseurs) et le terme en $\mathrm{C}_{2}$ (eux). L'interprétation de cet énoncé comme décrivant un 'accident' lors d'une chasse (tel n'est pas le cas a priori avec la construction préfixale : les chasseurs s'entre-tuent) tient précisément à cette fermeture ${ }^{11}$.

On voit qu'au lieu que I-E marque une neutralisation par rapport à la bipolarité première, I-E renvoie ici à une neutralisation au sein de la bipolarité.

Dans cet exemple avec se tuer, à cette fermeture va se rajouter la réciprocité. Dans un premier temps, à cause de se, l'élément $b$ (" entité ») non lexicalisé est récupéré par la mise en relation avec l'argument source, soit les chasseurs entre eux ${ }^{12}$; l'élément $a$ est, comme dans les exemples avec se tuer décrits ci-dessus, lexicalisé et correspond au GN en Co, donc à les chasseurs en tant que « déclencheur». Dans un deuxième temps, les chasseurs entre eux signifie qu'il y a une neutralisation dans la classe des chasseurs, alors qu'il y aurait pu y avoir un élément extérieur (ce qui aurait pu être ce que les chasseurs tuent). On a ainsi une double fermeture : les chasseurs sont tués par eux-mêmes et non par d'autres déclencheurs (tueurs) ; les chasseurs ne tuent pas à l'extérieur de la classe des chasseurs, et les tueurs de chasseur ne sont pas extérieurs aux chasseurs.

Nous donnons encore deux exemples :

11 Dans Ashino (2012), nous distinguons différents types de « fermeture ».

12 Rappelons que le terme source peut correspondre non seulement au référent d'un GN, mais aussi (et plus souvent) à un état ou une activité de ce référent (cf. 'Paul utilisant le revolver' en (19) ; 'Paul impliqué dans un accident de voiture' en (20) ; 'Paul travaillant' en (21)). 
(26) Dans la ville de banlieue de Little Stempington, les femmes au foyer ne tuent pas le temps, elles se tuent entre elles. (Libération)

(27) [=(14)] Le narcotrafic a gagné des batailles culturelles et médiatiques dans une société qui s'est protégée du problème en ayant recours au déni : «Les tueurs se tuent entre eux ». (Courrier International)

Dans l'un et l'autre exemples, l'altérité première vient de l'opposition entre I (ensemble) et $\mathbf{E}$ (élément extérieur) : I (les femmes au foyer) et $\mathbf{E}$ (le temps) en (26), I (les tueurs) et $\mathbf{E}$ (non lexicalisé, mais a priori 'les victimes') en (27); cette altérité est neutralisée par l'identification de $\mathbf{E}$ à $\mathbf{I}$.

En résumé, la construction prépositionnelle se tuer entre eux correspond à l'un des trois cas de neutralisation, celle-ci passant par la prise en compte, sur un mode effectif ou fictif, d'un terme extérieur (E) à un ensemble $(\mathbf{I})$, puis par la remise en cause de l'existence de ce terme par l'identification de $\mathbf{E}$ à $\mathbf{I}$, ce qui se traduit sur le plan formel par la coréférence entre le $\mathrm{N}$ en $\mathrm{Co}$ et le $\mathrm{N}$ en $\mathrm{C}_{2}$.

\section{5. s'entre-tuer comme " procès sans fin "}

\subsection{Les verbes préfixés comme "prédicats complexes"}

À la suite d'une série de travaux de D. Paillard $(2004 ; 2006$; 2007) consacrés à l'étude des verbes préfixés en russe et en français, nous considérons qu'un verbe préfixé peut être décrit comme un " prédicat complexe », produit de la combinatoire de deux prédicats : le verbe correspondant à la base d'une part, le préfixe assimilé à un prédicat $\mathbf{R}$ mettant en relation deux termes () et ()y, d'autre part.

Ci-dessous nous reprenons les points principaux qui ont été avancés dans Paillard (2007, p. 140):

- La combinatoire en question suppose que le verbe simple et le préfixe soient représentés de façon analogue ;

- Leur représentation articule deux niveaux : un niveau où est formulée leur identité sémantique par une forme schématique (FS) ; un niveau où ils sont représentés l'un et l'autre comme un prédicat avec ses arguments;

- Autant la forme schématique et le schéma prédicatif peuvent être très différents pour les verbes simples, autant la forme schématique et le schéma prédicatif du préfixe sont homologues. Cela tient au fait que la forme schématique de la préposition est déjà ternaire en elle-même avec deux arguments et une relation de repérage ; la forme schématique des bases verbales est beaucoup plus variable.

- Verbe et préfixe participent à la combinatoire par leur FS et par leur schéma prédicatif, ce qui peut se noter de la façon suivante :

$$
\begin{array}{llrl}
\mathbf{F S}(\text { verbe) }: a, b, c & \underline{\mathbf{e}} & \mathbf{F S} \text { (préfixe) }: \mathbf{X ~ R ~ Y ~} \\
<() \mathrm{r}()> & & <()_{\mathbf{x}} \mathrm{R}()_{\mathbf{y}}>
\end{array}
$$

Ce schéma appelle quelques commentaires :

$-a, b, c$ notent les éléments d'une FS donnée ;

- Il n'y a pas de correspondance simple entre l'élément de la FS et la place d'argument pour la base verbale ; 
- La combinatoire base-préfixe repose sur une relation de repérage (marquée par l'opérateur $\underline{\mathbf{c}}$ ) entre le verbe, élément repéré, et le préfixe, élément repère ;

- Cette opération de repérage s'interprète comme la reconstruction d'un élément $a$ de la FS du verbe dans l'espace du préfixe ;

- Cette reconstruction signifie de façon régulière que l'élément $a$ de la FS du verbe est pris comme $\mathbf{X}$ dans l'espace du préfixe. À ce titre, $\boldsymbol{a}$-X reçoit les déterminations dont $\mathbf{Y}$ est la source ;

- Par conséquent, contrairement à ce qui s'observe pour la préposition, avec $\mathbf{Y}$ correspondant au terme introduit par la préposition et $\mathbf{X}$ pouvant correspondre à différents arguments, pour le préfixe, $\mathbf{X}$ correspond régulièrement à l'un des éléments de la forme schématique de la base verbale, tandis que $\mathbf{Y}$ est plus variable et, en tous les cas, n’a pas a priori de réalisation lexicale.

\section{2. s'entre-tuer}

Pour illustrer le mécanisme de la préfixation de se tuer par entre-, nous proposons d'analyser la séquence les chasseurs s'entre-tuent, ce qui permettra de dégager des similitudes et différences entre les deux constructions en question.

L'élément $a$ (« déclencheur») de la FS de tuer est lexicalisé par le GN en Co : les chasseurs. L'élément $b$ («entité ») n'est pas lexicalisé, mais se pose qu'il est récupéré contextuellement par la mise en relation avec l'argument source qui est le seul terme pouvant tenir lieu d'« entité »; à la différence de la construction prépositionnelle, cet argument source est les chasseurs et non pas les chasseurs entre eux, puisqu'il n'y a pas de terme en $\mathrm{C}_{2}(e u x)$. L'élément $b$ correspond donc lui aussi à les chasseurs en tant que «victimes ».

Conformément au schéma de préfixation donné ci-dessus, c'est l'élément $a$ qui est pris comme $\mathbf{X}(\boldsymbol{a}-\mathbf{X})$ dans l'espace du préfixe : ce sont donc les 'chasseursdéclencheurs' (tueurs) qui se trouvent repérés sur le domaine associé à $\mathbf{Y}$ et celui-ci correspond au seul autre élément de la FS du verbe, à savoir l'élément $b$. Ce domaine associé à $\mathbf{Y}$ (domaine des chasseurs-victimes) est défini par entre- comme étant neutralisé $\mathbf{I}-\mathbf{E}$, au-delà de l'altérité première $\mathbf{I} \mid \mathbf{E}$. Avec entre- préfixe, $\mathbf{I}$ et $\mathbf{E}$ sont interprétables respectivement comme les $e_{1}$ et $e_{2}$ de la FS du verbe, à savoir pour les chasseurs-victimes 'être en vie' et 'être mort'; la configuration est donc différente de celle que l'on a vue dans le cas de se tuer entre eux où $\mathbf{I}$ correspond à un ensemble en Co et $\mathbf{E}$ à un terme extérieur à $\mathbf{I}$. La neutralisation $\mathbf{I}\left(e_{1}\right)-\mathbf{E}\left(e_{2}\right)$ s'interprète alors comme indétermination quant à savoir si, à l'issue du procès désigné par tuer, on reste en $e_{1}$ ('être en vie') ou on passe à $e_{2}$ ('être mort').

On voit qu'avec la construction préfixale, le verbe tuer est privé de sa transitivité forte, à la différence de ce qu'on observe avec la construction prépositionnelle où le passage de $e_{1}$ à $e_{2}$ dans lequel l'élément $b$ est pris est maintenu : s'entre-tuer désigne donc un 'événement hors télicité' (un événement qui n'est pas un achèvement); autrement dit, s'entre-tuer en tant que tel ne signifie pas que des sujets soient passés de 'vie' $\left(\mathbf{I}=e_{1}\right)$ à 'trépas' $\left(\mathbf{E}=e_{2}\right)$, parce que la neutralisation marquée par entre- préfixe renvoie à l'indétermination du rattachement de l'élément $b$ (les chasseurs en tant que 'victimes') soit à $\mathbf{I}$ soit à $\mathbf{E}$; ce qui a pour effet que, par là même, le passage à $\mathbf{E}$ est bloqué. 
Cela ne signifie pas pour autant que contextuellement ou situationnellement, il ne soit pas fait référence à des cadavres. D'une certaine façon, s'entre-tuer est 'en amont', comme le montrent les exemples suivants :

(28) Un gradé de la police entrant dans une pièce où deux ou plusieurs personnes gisent mortes sur le sol, déclare : «ils se sont entre-tués».

(29) Laissons-les s'entre-tuer.

La valeur réciproque avec s'entre-tuer quant à elle découle du fait que les chasseurs-victimes (b) étant identiques au $\mathrm{N}$ en Co, ils sont également «déclencheurs »; entre- signifie à la fois que les tueurs sont les 'à tuer' et qu'ils ne sont pas 'tués' (cf. le blocage du passage à $e_{2}$ ), mais 'en cours de tuerie'. Le procès réciproque désigné par s'entre-tuer est donc présenté comme étant 'sans issue / sans fin' - un procès qui tourne en rond.

\section{Retour sur les contraintes}

Les différences entre entre préposition et entre-préfixe que nous avons mises en évidence permettent de rendre compte des contraintes syntaxiques et des effets de sens sémantiques que nous avons mentionnés en 1.2. :

(i) L'impossibilité pour se tuer entre eux d'avoir les GN en Co désignés par tous en (6) et par on en (7) s'explique par le fait qu'on ne peut pas reconstruire un terme extérieur $(\mathbf{E})$ : tous, en tant que désignant la totalité, exclut tout terme extérieur ; on, en tant qu'indéfini, ne permet pas de distinguer un terme sur un ensemble d'individus (il et le), ce qui bloque toute altérité première.

(ii) La contrainte qui pèse sur la construction prépositionnelle avec un $\mathrm{N}$ duel en Co, coordonné (cf. (8), (9)) ou quantifié par le numéral deux (cf. (10)) tient à la difficulté de construire un domaine neutralisé uniquement à partir de deux éléments : les deux éléments sont nécessairement différenciés.

(iii) La construction préfixale (et non la construction prépositionnelle) permet une interprétation "métaphorique » de (se) tuer en (11) et (12) : entre- préfixe bloque le passage à $e_{2}$ ('mort'), ce qui est compatible avec la description du procès ('comme si c'était une tuerie' qui n'en est en fait pas une). Ce blocage du passage à $e_{2}$ explique également que s'entre-tuer ne soit pas un verbe d'achèvement (cf. (v)).

(iv) Les effets de sens particuliers associés à la construction prépositionnelle exprimant la « délimitation d'un groupe d'individus » ou marquant l'« opposition d'un groupe à un autre » (cf. Rivière \& Guentcheva (2007)) peuvent être considérés comme les deux phases de l'opération de la neutralisation construite par entre dans sa construction prépositionnelle : on a au départ une altérité première ("opposition ») entre I (ensemble) et $\mathbf{E}$ (terme extérieur à cet ensemble) ; cette altérité initiale est remise en cause par l'identification de $\mathbf{E}$ à $\mathbf{I}$, qui débouche sur la fermeture ("délimitation») de $\mathbf{I}$ relative au procès.

\section{Conclusion}


Dans cet article, nous avons voulu montrer que l'articulation variation / invariance des unités morpho-lexicales doit être pensée également pour une même unité fonctionnant dans deux structures syntaxiques différentes. L'unité entre(-) que nous avons étudiée doit être décrite comme renvoyant à deux opérations différentes de neutralisation selon les constructions dans lesquelles elle s'inscrit.

- Avec la construction prépositionnelle (se tuer entre eux), la neutralisation s'interprète comme la négation / la remise en cause de la différenciation première entre $\mathbf{I}$ (ensemble) et $\mathbf{E}$ (terme extérieur à $\mathbf{I}$, explicite ou non), au sens où $\mathbf{E}$ est identifié à I, ce qui signifie qu'il n'y a pas de $\mathbf{E}$ distinct de $\mathbf{I}$. Nous avons caractérisé ce type de neutralisation comme exprimant une « fermeture » de $\mathbf{I}$ relativement au procès.

- Avec la construction préfixale (s'entre-tuer) en revanche, la neutralisation s'interprète comme renvoyant à l'indétermination du rattachement de l'élément $b$ ('les chasseurs-victimes') à $\mathbf{I}$ ou à $\mathbf{E}$, I et $\mathbf{E}$ correspondant respectivement aux deux états $e_{1}$ et $e_{2}$ que mobilise la FS de tuer. D'où le blocage du passage à $e_{2}$ pour l'élément $b$. Ce type d'indifférenciation caractérise le procès comme étant « sans fin ».

\section{Bibliographie}

AsHinO, F., 2012, Contribution à l'étude de la notion de 'réciprocité' en français contemporain, Thèse de Doctorat, Université Paris VII.

BenetTi, L. \& HeYNA, F., 2006, «Topologie de la relation partie-tout dans les verbes préfixés en entre- et en dé- ", in Kleiber, G., Schnedecker, C. \& Theissen, A. (éds), La relation partie-tout, Louvain-Paris, Peeters, p. 611-631.

CAmus, R. \& DE VoGÜÉ, S. (dir.), 2004, Variation sémantique et syntaxique des unités lexicales : étude de six verbes français, LINX, 50, Université Paris X.

Culioli, A., 1990, "Formes schématiques et domaine », Pour une linguistique de l'énonciation, Tome 1, Paris, Ophrys, p. 115-126.

Culioli, A., 1999, "Qu'est-ce qu'un problème en linguistique ? Etude de quelques cas », Pour une linguistique de l'énonciation, Tome 3, Paris, Ophrys, p. 59-66.

DE VogüÉ, S., 2004, «Syntaxe, référence et identité du verbe filer », in Camus \& De Vogüé (eds), p. 135-167.

Franckel, J.-J. \& Paillard, D., 2007, Grammaire des prépositions, Tome 1, Paris, Ophrys.

PAILLARD, D., 1979, Voix et aspect en russe contemporain, Paris, Institut d'études slaves.

PAILlard, D., 2000, «A propos des verbes “polysémiques" - identité sémantique et principes de variation ", in Cordier, F., François, J. \& Victorri, B. (éds), Syntaxe et sémantique, 2, p. $99-120$

PAILLARD, D., 2004, « À propos des verbes préfixés », Slovo, 30-31, Inalco, p. 13-44.

PAILLARD, D., 2006, «A propos de verbes préfixés avec la base $K A Z$-», in Lebaud, D., Paulin, C. \& Ploog, K. (éds), Constructions verbales et production de sens, Presses Universitaires de Franche-Comté, p. 321-336.

PAILLARD, D., 2007, "Verbes préfixés et intensité en russe et en français », Travaux de linguistique, 55, p. 133-149. 
ENTRE, entre préposition et préfixe

PAuly, E., 2010, La polysémie - Réflexions théoriques, méthodologiques et application à la lexicographie : l'exemple des verbes aller, partir et tirer en français contemporain, Paris, L'Harmattan.

VANDELOISE, C., 1986, L'espace en français, Paris, Seuil. 